\title{
ROLE OF SERUM FERRITIN AND SERUM AMINOTRANSFERASES IN PREDICTING THE SEVERITY IN DENGUE FEVER WITH THROMBOCYTOPENIA
}

\author{
Diwakar TN1, Madhu $G^{2}$
}

${ }_{1}^{1}$ Associate Professor, Department of General Medicine, Bangalore Medical College and Research Institute, Bangalore, Karnataka, India. 2Postgraduate Student, Department of General Medicine, Bangalore Medical College and Research Institute, Bangalore, Karnataka, India.

\section{ABSTRACT}

\section{BACKGROUND}

Dengue fever is an important cause of febrile illness in the tropical and subtropical areas and over 2.5 billion people, over $40 \%$ of the world population are now at risk from dengue ${ }^{1}$. WHO currently estimates that there may be $50-100$ million dengue infections worldwide every year. ${ }^{1}$ In India between 2015 and 2017, out of 4,17,480 cases, 790 deaths have been reported according NVBDCP data. ${ }^{2}$ This study aims at determining the role of serum ferritin and serum aminotransferases in predicting severity of dengue patients with thrombocytopenia.

\section{MATERIALS AND METHODS}

It is a cross sectional observational study conducted on 100 patients. Patients were assessed by clinical WHO criteria and were investigated for severity of dengue. Severity of dengue and its association with serum ferritin and serum aminotransferase were analysed using student ' $t$ ' test and ROC curve was plotted to find the sensitivity and specificity.

\section{RESULTS}

In our study conducted on 100 patients, the age distribution was between 18 yrs. to 65 yrs. with mean age of $32 \pm 10$ yrs. Male to female ratio was 69:31. Out of 100 patients, 28 patients had severe dengue as per the WHO 2009 criteria. Significant association was found between raised serum ferritin, AST, ALT and severe dengue ( $p<0.001)$. ROC curve shows that AST with best cut-off of 380 IU/L predicts the severity of dengue with sensitivity of $82.1 \%$ and specificity of $94.4 \%$, followed by ALT (Sensitivity $82.1 \%$ and specificity $93.1 \%$ ) and serum ferritin (Sensitivity $85.7 \%$ and specificity $87.5 \%$ ) respectively.

\section{CONCLUSION}

Results of this study shows significant association between raised AST, ALT, serum ferritin and severity of Dengue and these parameters may serve as a potential biomarker in early prediction of severe dengue.

\section{KEY WORDS}

Dengue, Acute Phase Reactant.

HOW TO CITE THIS ARTICLE: Diwakar TN, Madhu G. Role of serum ferritin and serum aminotransferases in predicting the severity in dengue fever with thrombocytopenia. J. Evolution Med. Dent. Sci. 2018;7(50):5378-5382, DOI: 10.14260/jemds/2018/1190

\section{BACKGROUND}

Dengue fever is viral fever caused by Dengue Virus (DENV) which belongs to genus flavivirus of Flaviviridae family. It is caused by one among the 4 subtypes of dengue virus (DENV1, DENV2, DENV3, and DENV4). Characteristics features are fever and constitutional symptoms and in severe cases shock and bleeding manifestations (DSS/DHF). ${ }^{3}$

Etiological diagnosis can be confirmed by serological testing and virus detection by isolation or molecular technique from the blood during the early febrile phase. Serological testing includes detection of NS1, IgM or IgG ELISA. ${ }^{3}$

The disease is considered to be hyper endemic in tropical and sub-tropical regions, most prevalent in Southeast Asia, the Americas, and Western Pacific regions. ${ }^{4}$

'Financial or Other Competing Interest': None. Submission 06-10-2018, Peer Review 25-11-2018,

Acceptance 03-12-2018, Published 10-12-2018.

Corresponding Author:

Dr. Diwakar Tumkur Narasimhamurthy,

No. 33, Haripriya, $6^{\text {th }}$ Cross, A-Sector, Amruthnagar,

Bangalore-560092, Karnataka, India.

E-mail: dr.diwakartn@gmail.com

DOI: $10.14260 /$ jemds $/ 2018 / 1190$

\section{(c) $($ ) $९$}

The first case of dengue was reported in Calcutta, India in 1945; India's first dengue fever epidemic was reported in 1963. The first major epidemic of DHF/DSS was identified in India in 1996.5

Based on trends in endemicity, India is identified as a Category B country as the country is reported to experience cyclic epidemics. ${ }^{6}$

Apart from routine laboratory investigations have been studied in the past to determine the tests that can be used to assess severity of dengue infection. Two such tests being serum aminotransferases and serum ferritin. ${ }^{7}$

Ferritin is an acute phase reactant and highly expressed by cells of the reticulo-endothelial system in response to infection and inflammation. Hyperferritinaemia is due to extensive immune activation seen in severe dengue. ${ }^{7}$

Elevation of the liver enzymes aspartate aminotransferase (AST) and alanine aminotransferase (ALT) is common in acute dengue illness, occurring in $65-97 \%$ of dengue patients, peaking during the convalescent period of illness (days 7-10). In dengue-endemic countries, dengue is an important cause of acute viral hepatitis. Elevated AST and ALT levels have been associated with bleeding and dengue haemorrhagic fever (DHF). ${ }^{8}$ 
This study aims at determining role of serum ferritin and serum transaminases in predicting the severity of dengue and also the correlation between the two tests.

\section{Aim of the Study}

The aim of the study was to determine the role of serum ferritin and serum aminotransferases predicting severity in dengue patients with thrombocytopenia.

\section{MATERIALS AND METHODS}

Those patients who has given an informed written consent and admitted as confirmed cases of dengue with thrombocytopenia in the hospital attached to BMCRI, during April 2018 to September 2018. The study protocol was approved by the BMCRI ethical committee.

\section{Inclusion Criteria}

1. Subjects who are detected with NS1 antigen or positive IgM antibodies against dengue virus

2. Age more than 18 years

3. Thrombocytopenia $(<1,50,000) \cdot{ }^{9}$

\section{Exclusion Criteria}

1. Patients with pre-existing liver disease.

2. Patients with Anaemia, iron overload state

3. Other causes of Thrombocytopenia and raised ferritin.

Serum ferritin levels and serum transaminase levels of all patients are measured on the day of admission. Patients will be followed up during their hospital stay and assessed the severe of Dengue. Detailed history, physical examination and necessary investigations were undertaken. Severity will be graded according to WHO classification. ${ }^{10}$

\section{Statistical Analysis}

The quantitative variables were expressed as mean \pm SD, while categorical variables were expressed in percentage. For comparison of two groups, Mann Whitney $\mathrm{U}$ test was used. $\mathrm{P}$ value $<0.05$ was considered as statistically significant with 95\% CI. ROC curve plotted to know the sensitivity and specificity of Serum ferritin, serum Aminotransferase to predict the severe dengue.

\section{RESULTS}

The sample size in our study was 100 patients. The age distribution was between 18 to 65 yrs. with mean age of patients being $32.03 \pm 11.9$ yrs. (Table 1). 69 were male and 31 are female (Fig. 1).

Among 100 patients, 28 patients diagnosed as severe Dengue according to WHO 2009 classification (Fig. 2). Among 28 patients diagnosed as severe Dengue, most of the patients developed more than 1 manifestation of severe dengue, 24 patients had bleeding manifestation, 14 patients had shock, 13 patients had organ dysfunction, 5 had altered mental status, 5 had respiratory distress (Fig. 3). Among organ dysfunction 8 patients had Hepatitis, 2 patients had AKI and 3 patients had both AKI and Hepatitis (Fig. 4)

\begin{tabular}{|c|c|c|c|c|}
\hline & Minimum & Maximum & Median & $\begin{array}{c}\text { Interquartile } \\
\text { Range }\end{array}$ \\
\hline Age & 18 & 65 & 29 & 14 \\
\hline
\end{tabular}

\begin{tabular}{|c|c|}
\hline Age & Number of Patients \\
\hline $18-25$ & 39 \\
\hline $26-35$ & 30 \\
\hline $36-45$ & 12 \\
\hline $46-55$ & 7 \\
\hline $56-65$ & 7 \\
\hline \multicolumn{2}{|c|}{ Table 1. Age Distribution of Patients Studied } \\
\hline
\end{tabular}

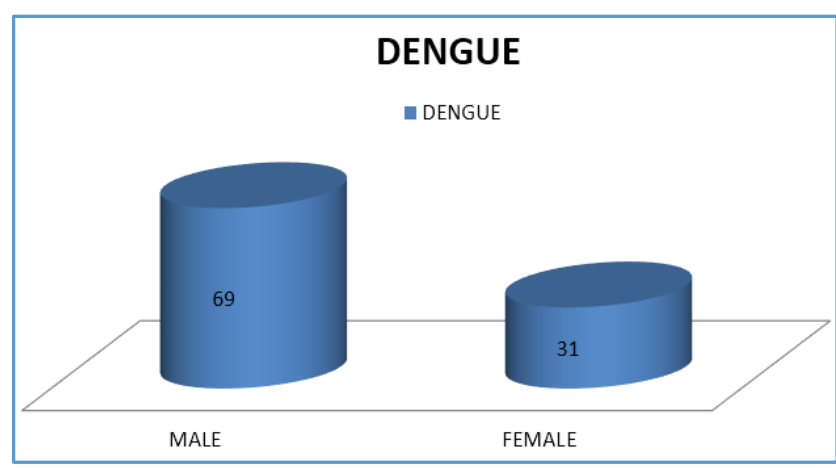

Figure 1. Gender Distribution of Patients Studied

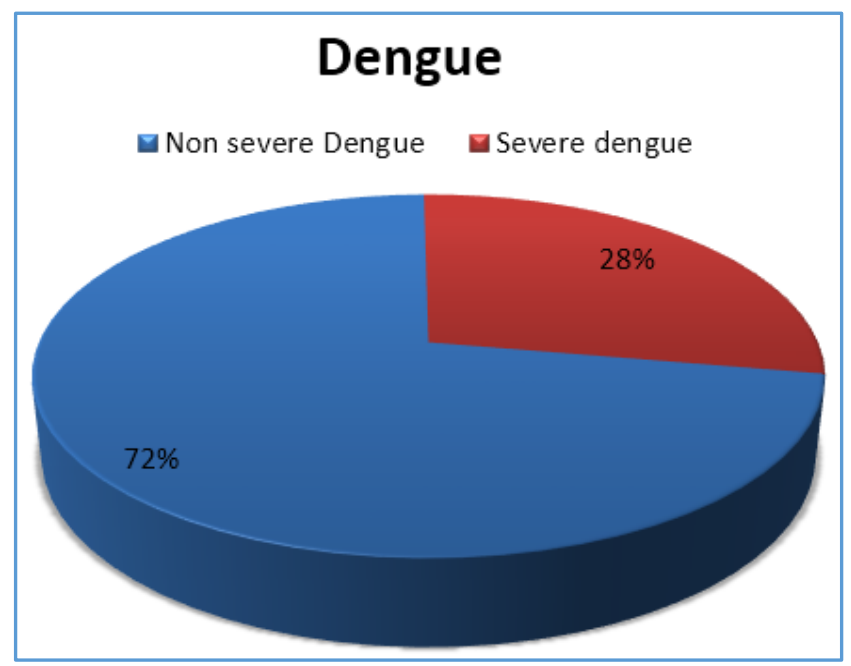

Figure 2. Patients Classified according to Severity

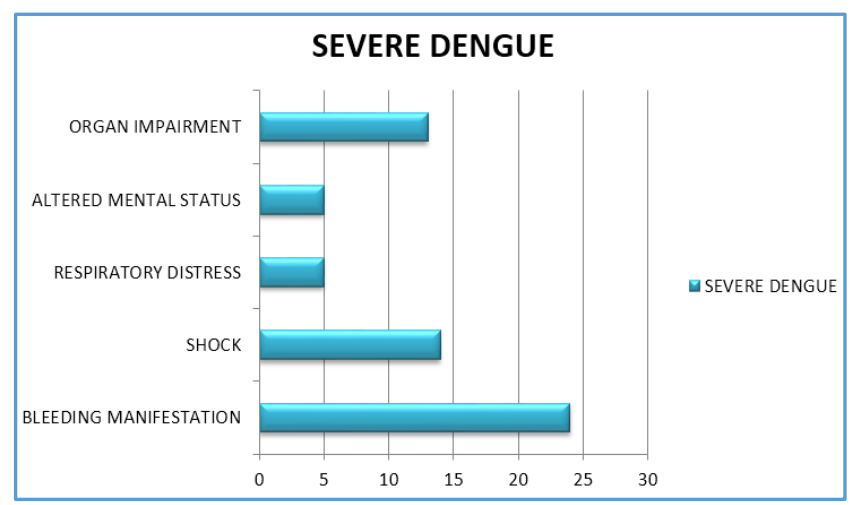

Figure 3. Manifestations of Severe Dengue 


\section{ORGAN DYSFUNCTION}

$\square$ HEPATITIS $\square$ AKI $\square \mathrm{AKI}+$ HEPATITIS

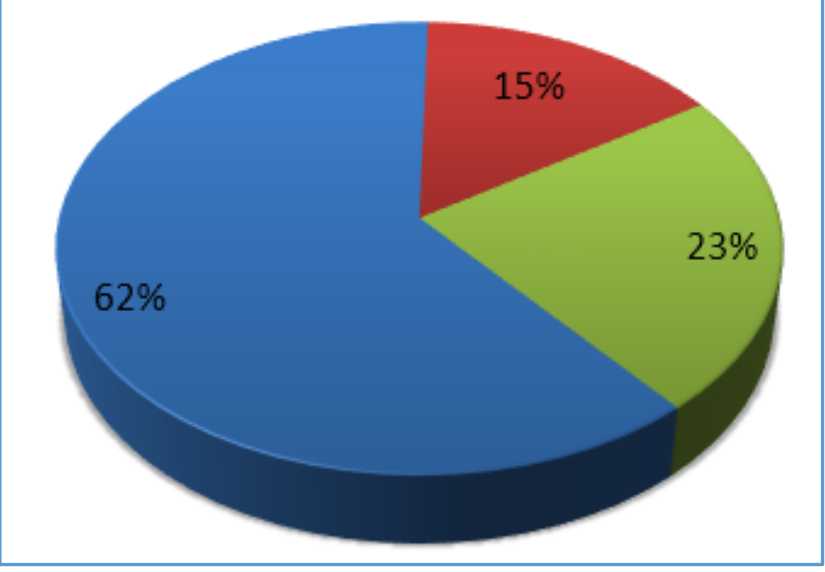

Figure 4. Pattern of Organ Dysfunction

Serum levels of Ferritin, AST and ALT were estimated in all Dengue patients with thrombocytopenia and the levels of individual parameter between severe and non-severe Dengue are shown in table 2 . Serum levels of each parameter in severe and non-severe dengue are compared using Mann Whitney U test. Severe dengue patients had higher levels of serum Ferritin, AST and ALT compared to non-severe dengue and statistically significant difference was observed between the groups ( $p<0.001$ ) (Fig. 5)

\begin{tabular}{|c|c|c|c|c|c|c|}
\hline & 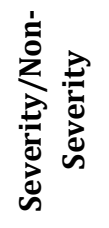 & $z$ & 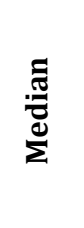 & 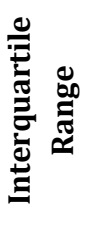 & 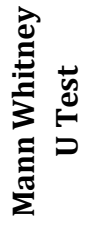 & 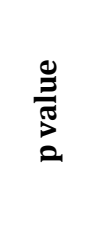 \\
\hline \multirow[t]{2}{*}{ Ferritin } & $\begin{array}{l}\text { Non- } \\
\text { Severe }\end{array}$ & 72 & 770 & 800 & \multirow[t]{2}{*}{194.5} & \multirow[t]{2}{*}{$<0.001$} \\
\hline & Severe & 28 & 2130 & 480 & & \\
\hline \multirow[t]{2}{*}{ SGOT } & $\begin{array}{l}\text { Non- } \\
\text { Severe }\end{array}$ & 72 & 64 & 116 & \multirow[t]{2}{*}{104} & \multirow[t]{2}{*}{$<0.001$} \\
\hline & Severe & 28 & 675 & 543 & & \\
\hline \multirow[t]{2}{*}{ SGPT } & $\begin{array}{c}\text { Non- } \\
\text { Severe }\end{array}$ & 72 & 36 & 102 & \multirow[t]{2}{*}{120} & \multirow[t]{2}{*}{$<0.001$} \\
\hline & Severe & 28 & 451 & 238 & & \\
\hline
\end{tabular}

Table 2. Comparison of Serum Levels of Ferritin, AST and ALT between the Groups

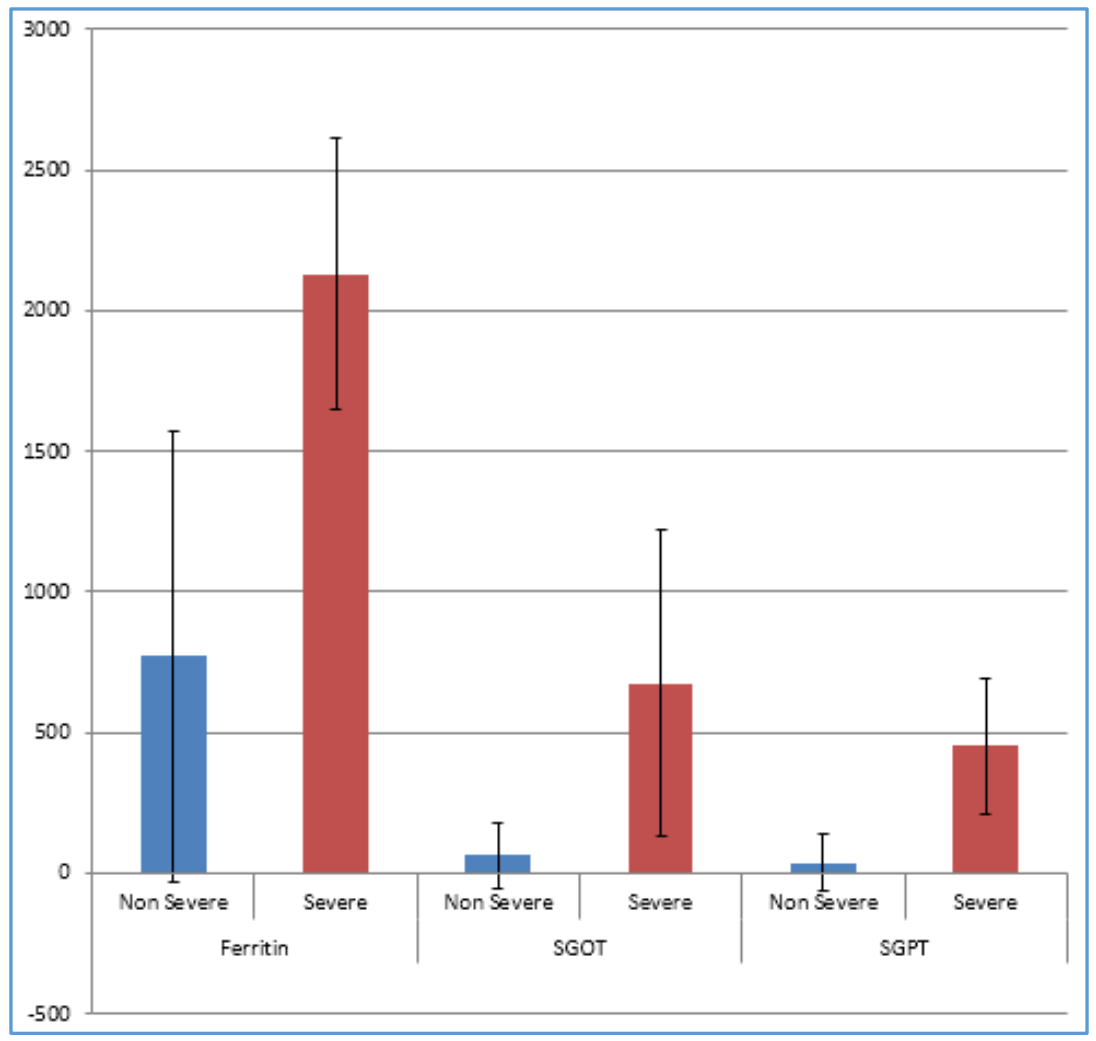

Figure 5. Statistical Differences of S. Ferritin, AST and ALT between Severe and Non-Severe Dengue Group

Does a threshold serum Ferritin, AST or ALT value defining severe dengue exist?

The levels of each parameter between 2 groups were subjected to ROC analysis to determine the reliability of serum Ferritin, AST and ALT value in defining the dengue severity (Fig. 6).
The value which had a maximum AOC (Youden Index) was taken has best for predicting the severity of dengue. Among all the 3 parameter AST topped with AUC 0.948 (95\% CI: 0.907 0.990 ) with best cut off value of $380 \mathrm{IU} / \mathrm{L}$ ( $\mathrm{p}$ value $<0.001$ ). The elevation of AST in severe dengue compared to non-severe dengue could predict the disease severity with a sensitivity of $82.1 \%$ and specificity of $94.4 \%$. (Table 3 ). 
The next best parameter was ALT with AUC 0.940 (95\% CI: 0.895-0.986) which can predict the severity with sensitivity of $82.1 \%$ and specificity of $93.1 \%$ with best cut off value of 270 $\mathrm{IU} / \mathrm{L}$ ( $\mathrm{p}$ value $<0.001$ ).

The last parameter was serum Ferritin with AUC 0.904 (95\% CI: 0.839-0.968) which can predict the severity with sensitivity of $85.7 \%$ and specificity of $87.5 \%$ with best cut-off of $1560 \mathrm{ng} / \mathrm{dl}$ ( $\mathrm{p}$ value <0.001).

Thus, the difference in AST between severe and non-severe dengue help in predicting the severity of disease with good sensitivity and specificity which was followed by ALT and serum Ferritin respectively.

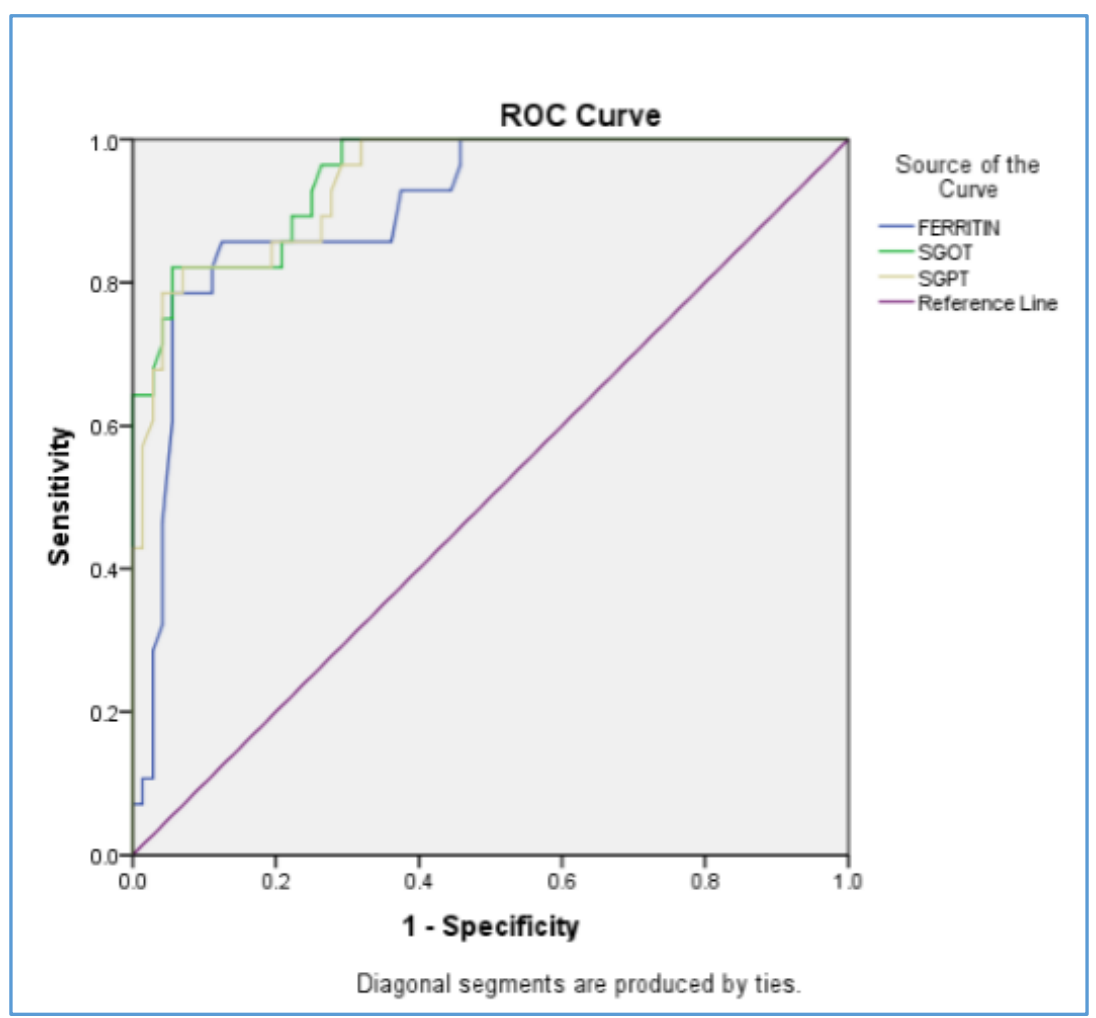

Figure 6. Receiver-Operating Characteristic Curves of 3 Parameters for Predicting Severe Dengue

\begin{tabular}{|c|c|c|c|c|c|c|c|c|}
\hline \multicolumn{6}{|c|}{ Area Under the Curve } & \multirow{3}{*}{ Cut-Offs } & \multirow{3}{*}{ Sensitivity (\%) } & \multirow{3}{*}{ Specificity (\%) } \\
\hline \multirow{2}{*}{$\begin{array}{l}\text { Test Result } \\
\text { Variable (s) }\end{array}$} & \multirow[t]{2}{*}{ Area } & \multirow{2}{*}{$\begin{array}{l}\text { Std. } \\
\text { Errora }\end{array}$} & \multirow{2}{*}{ Asymptotic Sig.b } & \multicolumn{2}{|c|}{$\begin{array}{c}\text { Asymptotic } 95 \% \\
\text { Confidence Interval }\end{array}$} & & & \\
\hline & & & & $\begin{array}{l}\text { Lower } \\
\text { Bound }\end{array}$ & $\begin{array}{l}\text { Upper } \\
\text { Bound }\end{array}$ & & & \\
\hline Ferritin & .904 & .033 & .000 & .839 & .968 & 1560 & 85.7 & 87.5 \\
\hline SGOT & .948 & .021 & .000 & .907 & .990 & 380 & 82.1 & 94.4 \\
\hline SGPT & .940 & .023 & .000 & .895 & .986 & 270 & 82.1 & 93.1 \\
\hline \multicolumn{9}{|c|}{ Table 3. Comparison of 3 Parameters } \\
\hline
\end{tabular}

\section{DISCUSSION}

Since the 1990s, epidemics of dengue have become more frequent in many parts of India. Over the period (2010-2014), $2,13,607$ cases (Incidence: 34.81 per million population) were reported11.During a more recent period (2015-2017) 4, 17, 480 cases and 790 death due to dengue fever were observed, in Karnataka in the year of 2017 alone 17,844 cases on dengue fever and 10 death due to dengue were reported. ${ }^{2}$

There are several studies in the past in dengue infected patients regarding the early predictor of severe dengue. These indicators appear in the plasma before the appearance of symptoms of severe dengue and help in the prognosis. In our study we tried to find the role serum ferritin and aminotransferases as a early predictor of severe dengue.

We observed in our study that 28 cases were diagnosed has severe dengue out of 100 . Most common manifestation of severe dengue was bleeding and most common organ involved is Liver. Our study shows significant association between raised serum ferritin $(p<0.001)$, AST $(p<0.001)$ and ALT $(p<0.001)$ in predicting severity of dengue.

This study shows AST predict the disease severity with a better sensitivity and specificity compared to another parameter. The next best parameter was ALT and S ferritin respectively.

Study by Soundravally $\mathrm{R}$ et al, concluded that raised ferritin levels could predict the dengue severity with sensitivity of $76.9 \%$ and specificity of $83.3 \%$ on the day of admission. ${ }^{12}$

Study conducted by Xiao-Jun Wang et al, observed that the abnormal level of AST occurs with the highest proportion of $80 \%$ in DHF and $75 \%$ in DF, followed by ALT with $54 \%$ in DHF and $52 \%$ in DF, respectively and concluded that AST and ALT may be used as indicators for the liver injury as well as for diagnosis and treatment effect in dengue infection. ${ }^{13}$ 
But, Linda $\mathrm{K}$ Lee et al. In 'Clinical Relevance and Discriminatory Value of Elevated Liver Aminotransferase Levels for Dengue Severity' concluded that although aminotransferase levels increased in conjunction with dengue severity, AST or ALT values did not discriminate between DF and DHF or non-severe and severe dengue. ${ }^{8}$

The limitations of our study are, it's a cross sectional observational study, $\mathrm{S}$ ferritin and aminotransferases are measured only on the day of admission Thus, a relatively larger cohort study with follow ups would require to ascertain the findings of the present study.

\section{CONCLUSION}

This study conducted on 100 patients, 28 patients had severe dengue. Significant association was found between raised AST ( $p$ value $<0.001$ ) and ALT ( $p$ value $<0.001$ ), ferritin ( $p$ value $<$ 0.001 ) and severity of dengue. Based on the highest specificity and sensitivity in predicting severe dengue cases AST $>$ ALT > serum ferritin can be used as indicators for an early prediction of severity of dengue.

\section{REFERENCES}

[1] www.searo.who.int/entity/vector_borne_tropical_dis ease/data/data_factsheet/en/

[2] http://www.nvbdcp.gov.in/index4.php?lang=1\&level $=0 \&$ linkid $=431 \&$ lid $=3715$

[3] Nimmannitya S. Dengue and Dengue hemorrhagic fever. In: Cook GC, Zumla AI, eds. Manson's Tropical diseases. 22nd edn. Edinburgh: Saunders Elsevier 2009.

[4] Fullerton LM, Dickin SK, Wallace SCJ. Mapping Global Vulnerability to Dengue using the Water Associated Disease Index. United Nations University. 2014.
[5] Shepard DS, Halasa YA, Tyagi BK, et al. Economic and disease burden of Dengue illness in India. Am J Trop Med Hyg 2014;91(6):1235-42.

[6] Amarasinghe A, Bhola AK, Halstead SB, et al. Uncovering Dengue in India: morbidity estimates. Global Journal of Medicine and Public Health 2014;3(3).

[7] Van de Weg CAM, Huits RM, Pannuti CS, et al. Hyperferritinaemia in Dengue virus infected patients is associated with immune activation and coagulation disturbances. PLoS Negl Trop Dis 2014;8(10):e3214.

[8] Lee LK, Gan VC, Lee VJ, et al. Clinical relevance and discriminatory value of elevated liver aminotransferase levels for Dengue severity. PLoS Negl Trop Dis 2012;6(6):e1676.

[9] Konkle BA. Disorders of platelets and vessel wall. In: Kasper DL, Fauci AS, Hauser S, et al. eds. Harrison's principles of internal medicine. 19 $9^{\text {th }}$ edn. NewYork: McGraw-Hill Education 2015.

[10] Nathan B, Renu DD, Maria G. Dengue guidelines for diagnosis, treatment, prevention and control. World Health Organization, 2009 edition.

[11] Mutheneni SR, Morse AP, Caminade C, et al. Dengue burden in India: recent trends and importance of climatic parameters. Emerging Microbes \& Infections 2017;6(8):e70.

[12] Soundravally R, Agieshkumar B, Daisy M, et al. Ferritin levels predict severe dengue. Infection 2015;43(1):13-9.

[13] Wang XJ, Wei HX, Jiang SC, et al. Evaluation of aminotransferase abnormality in dengue patients: a meta-analysis. Acta Trop 2016;156:130-6. 